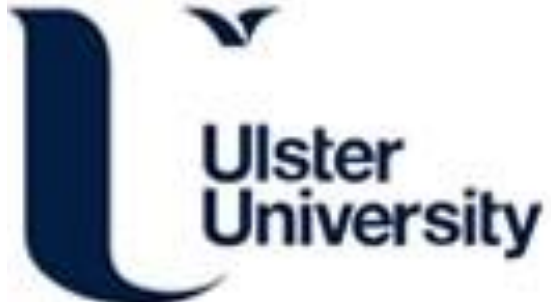

Accessing Tele-Services using a Hybrid BCl Approach

Brennan, CP., McCullagh, PJ., Lightbody, G., Galway, L., Feuser, D., \& Martin, S. (2014). Accessing TeleServices using a Hybrid BCl Approach. In Unknown Host Publication (Vol. LNCS, pp. 110-123). Springer. https://doi.org/10.1007/978-3-319-19258-1_10

Link to publication record in Ulster University Research Portal

\section{Published in:}

Unknown Host Publication

Publication Status:

Published (in print/issue): 10/06/2014

DOI:

10.1007/978-3-319-19258-1_10

\section{Document Version}

Publisher's PDF, also known as Version of record

\section{General rights}

Copyright for the publications made accessible via Ulster University's Research Portal is retained by the author(s) and / or other copyright owners and it is a condition of accessing these publications that users recognise and abide by the legal requirements associated with these rights.

\section{Take down policy}

The Research Portal is Ulster University's institutional repository that provides access to Ulster's research outputs. Every effort has been made to ensure that content in the Research Portal does not infringe any person's rights, or applicable UK laws. If you discover content in the Research Portal that you believe breaches copyright or violates any law, please contact pure-support@ulster.ac.uk. 


\title{
Accessing Tele-Services using a Hybrid BCI Approach
}

\author{
Chris Brennan ${ }^{1}$, Paul McCullagh ${ }^{1}$, Gaye Lightbody ${ }^{1}$, Leo Galway ${ }^{1}$, \\ Diana Feuser ${ }^{2}$, José Luis González ${ }^{3}$, and Suzanne Martin ${ }^{4}$ \\ 1 Computer Science Research Institute, Ulster University, UK, BT37 0QB \\ \{brennan-c15\}@email.ulster.ac.uk; \{p.j.mccullagh\}@ulster.ac.uk \\ http://www. compeng.ulster.ac.uk/csri.php \\ 2 Institute of Automation, University of Bremen, Otto-Hahn-Allee 1, 28359 Bremen, \\ Germany \{diana.feuser\}@uni-bremen.de \\ 3 Telefonica de España, Ronda de la Comunicación, s/n, 28050 Madrid, Spain \\ ${ }^{4}$ Institute of Nursing and Health Research, Ulster University, UK, BT37 0QB
}

\begin{abstract}
Brain Computer Interface (BCI) technology has achieved limited success outside of laboratory conditions. This technology is hindered by practical considerations of set up, lack of robustness and low Information Transfer Rate (ITR). There are two interfaces in a BCI system: the brain's interface with the computer and the computer-environment interface, which provides access to applications for the user. Three user services were implemented: control of the smart home, entertainment and communication. These may be accessed through a graphical user interface controlled by a BCI. The paper contrasts the performance of an SSVEP based system with a hybrid BCI comprising eye gaze and muscle response (measured at the scalp). The hybrid developed utilizes the EPOC for recording electrical potential and an EyeTribe gaze tracker; these can be combined to provide more robust interaction with applications. Average ITR for the eye tracker and hybrid approaches (190-200 $\mathrm{bpm}$ ) are higher than for our SSVEP approach (approx. $15 \mathrm{bpm}$ ), for the same applications. The poor performance of our SSVEP system was due to the temporal duration of the stimulation $(7 \mathrm{~s})$ and partly because not all participants could achieve an accuracy of greater than $50 \%$. The current challenge is the replacement of the scalp recorded muscle component with a reliable user modifiable EEG measure.
\end{abstract}

Keywords: Applications, Brain-Computer Interface (BCI), Communication, Control, Entertainment, Eye-tracking, hBCI, Hybrid

\section{Introduction}

The Brain-Computer Interface (BCI) is a paradigm that aims to empower a user's capabilities by providing an input modality to the computer that does not require the involvement of the user's peripheral nerves and muscles [1]. In recent years, our increased understanding of human brain function, coupled with advances in technology, has led to significant advances in BCI technology. In 
particular signal processing algorithms utilize specific patterns in brain activity across both spatial and temporal domains and translate these into classified commands for action by the computer. Increasingly as the algorithms become more complex, the resulting systems may be endowed with more intelligent, contextual processing, thereby leading to augmentation for the user.

The most commonly employed BCI approach, Electroencephalogram (EEG), measures electrical activity recorded along the scalp, resulting from ionic current flows within the neurons of the brain [2]. In this case, the mental state of the user is probed to generate the brain activity patterns that facilitate control, which will vary in accordance with the operating protocol [3]. The most commonly employed approaches include the P300 component of Event-Related Potential (ERP), Event-Related Desynchronisation/Synchronisation (ERD/ERS), motor potentials, Steady State Visually Evoked Potentials (SSVEP) and Slow Cortical Potentials (SCP) [4]. Current limitations include slow Information Transfer Rates (ITR), high inter/intra-subject variability, inconvenient set up procedures, and the need for carefully controlled environments [5]. Such restrictions adversely affect those that could potentially benefit most from this technology, individuals who suffer from disease and traumatic brain injury [6].

To compensate for these limitations and utilize the advantages of different BCI methods, recent emphasis has been placed upon 'hybrid' architectures [7]. One possible architecture may combine BCI approaches (e.g. motor imagery and sensory stimulation) with separate technologies, such as eye tracking [6]. Hybrid BCIs (hBCI) have the potential to increase transfer rates and classification accuracy, thereby promoting the acceptance and adoption of the technology. An hBCI, which combines EEG with eye tracking, may employ inputs sequentially or collaboratively, with each modality responsible for distinct aspects of user input processing or for cooperative command classification [6]. As conventional BCI systems are hindered by an extensive list of limitations, which have prevented exploitation, a hybrid BCI has the potential to overcome these and promote widespread deployment. Such advances could see this technology being utilised in other application areas such as entertainment, neurorehabilitation, neuroprosthetics, immersive education, and other specialist areas. By improving performance and usability through hybrid implementations, advances in other areas can be made, resulting in improvements to the technology overall, which will cycle back to and improve conventional BCI's as an assistive technology for the severely disabled. Current P300 systems, which are used by individuals that have severe mobility reducing conditions such as ALS, can achieve a spelling rate of between 5-10 characters per minute. For some people this is their only means of communication and so, it is of importance to improve upon this technology, thus improving social inclusion, quality of life, and well-being.

This work supports the hypothesis that a hybrid sensor fusion approach will facilitate better human-computer interaction because performance and utility will be improved. An architecture initially developed for the EU FP7 BRAIN project [8] has been extended to facilitate an hBCI approach. The architecture consisted of a set of tools for interface development and testing, along 
with SSVEP and ERD/ERS recording. An Intuitive Graphical User Interface (IGUI) tool has been extended to support the combination of eye tracking and pseudo-EEG control through the use of inexpensive, easily deployed devices. It is anticipated that the use of commercially available technology will facilitate better human-computer interaction within the smart environment, permitting access to a number of computer-mediated services. Three trial user services were implemented: entertainment, control of the environment, and communication by iconography.

\section{$2 \quad$ Architecture and Interfaces}

In BRAIN the BCI pipeline comprised data acquisition, signal processing (BCI2000), and user interface control using classified UDP packets. It has since been updated to receive input from multiple input modalities, see Fig 1 . The Intuitive User Interface (IUI) is realized as two components; the user interface itself and a programmatic application interface, known as the Universal Application Interface (UAI). The IGUI provides a user interface that interacts with a virtual representation of a domestic environment, providing a BCI channel for domotic control, entertainment, and communication. The IGUI, implemented using the Java programming language, permits operating system independent deployment and provides an interactive menu system that has been specifically structured to provide four-way directional control. Each menu item controls an event that can be triggered within the smart environment.

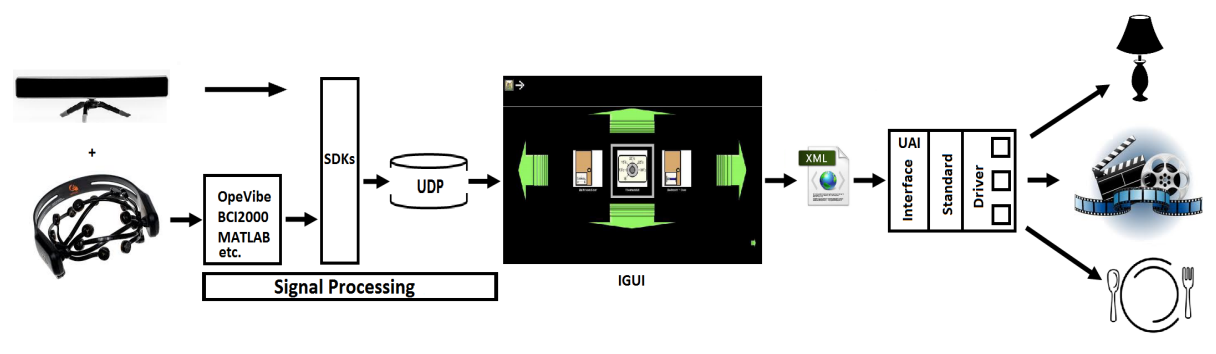

Fig. 1. The pipeline components of the BCI and ET collaborative system for domotic, entertainment and communication control.

The UAI interacts with the BCI device through the IGUI for the purpose of providing access to entertainment and communication packages as well as domotic device control. Device interaction may implement a relevant interface such as: X10, Universal Plug and Play (UPnP). UPnP was selected as the communication protocol used in middleware layer to provide interoperable specification with common protocols to other technologies, offering the possibility of wrapping other technologies. The architecture offers pervasive peer-to-peer network 
connectivity of PCs, intelligent appliances, and wireless devices. The UPnP architecture is a distributed, open networking architecture that uses TCP/IP and HTTP to control data transfer among networked devices in the home. The design aim was to provide an easily extensible interface that could support various input modalities (BCI paradigms, eye tracking, etc.). The UAI is the second component of the IUI and is responsible for providing a generic platform that forms the bridge between the BCI platform, IGUI, and applications and devices. This interface also facilitates leisure activities such as painting with the users brain waves [9].

\section{Control of the IGUI}

The first phase of experimentation incorporated a series of preliminary tests, which aimed to evaluate the IGUIs performance in terms of quality assurance. In the first experiment, the IGUI was controlled by features classified from EEG; recorded using the TMSi amplifier, Porti7, and EasyCap combination. Both devices are research or medical-grade products which, when coupled together, provide high quality EEG acquisition. The sampling frequency for the Porti7 amplifier was set to $256 \mathrm{~Hz}$ and the electrodes were placed at positions $\mathrm{PZ}, \mathrm{PO} 3$, PO4, OZ, O9, O10 and referenced at site AFZ.

For the BCI paradigm, SSVEP was utilized as the control mechanism and calibration was handled by the BCI-Wizard, which facilitates both frequency and threshold calibration. The calculation of a threshold was computed in BCI2000 for each calibrated or selected frequency. As a quality criterion, an Area Under ROC Curve (AUC) was calculated and if the AUC exceeded a predefined threshold, the calibration was regarded as suitable for further use. The SSVEP signal processing approach takes advantage of the Minimum Energy Combination (MEC) method to estimate power changes in the acquired EEG signals and classify the signal with the highest signal-to-noise ratio (SNR) if the predetermined threshold was exceeded. This algorithm, implemented in $\mathrm{C}++$ as a BCI2000 signal processing module, commissions a spatial filter that readjusts the input channels in order to minimize interference. As a result, electrodes that transmitted poor signals were ignored. Moreover, the combination matrix is constantly adapted to signal changes over time, which is executed on-line in BCI2000. Classification was always based on a two second sliding window and signals were processed every $125 \mathrm{~ms}$.

Stimulation was provided by four Light Emitting Diodes (LEDs) mounted around the outside of a visual interface. Each LED corresponded to a specific direction, left, up, right, down and flickered at $13 \mathrm{~Hz}, 14 \mathrm{~Hz}, 15 \mathrm{~Hz}$ and $16 \mathrm{~Hz}$ respectively. The user was required to make a cognitive link between a particular direction and the corresponding LED. For example, to move left the user had to focus on the LED located to the left of the display. This method of control was restrictive, due to the location of the LEDs. When navigating around the menu, the user did not have a full view of the changes they were affecting. In addition to the physical constraints, some users found this as a cognitive challenge since the 
directional command arrows and manipulation of menu items caused confusion and difficulties when attempting to navigate to a specific item. Nevertheless, for a control group of five users ( $\mathrm{N}=5$ ), the mean accuracy achieved was $69.6 \%$ $\pm 32.9 \%$. However, as a method for measuring the minimum level of tolerated accuracy is yet to be established within the BCI community, we contrasted the achieved results by conducting a BCI accessibility assessment, which provided an indication of the minimum level of accuracy for each user. The individual accuracy values are further expanded in Table 1.

Table 1. Five subjects participated in the BCI accessibility assessment and then completed the command operation assessment. The results, shown in this table, contrast user specific accuracy levels and the levels achieved using a BCI application. Desirable accuracy is the minimum level of control, which a subject deems to be satisfactory and the Acceptable Accuracy is minimum level of control before being considered unusable [10]

\begin{tabular}{llll}
\hline User & Desirable Acc. \% & Acceptable Acc. \% & Achieved Acc. \% \\
\hline 1 & 79 & 81 & 16 \\
2 & 77 & 81 & 95 \\
3 & 77 & 76 & 62 \\
4 & 74 & 77 & 80 \\
5 & 78 & 77 & 95 \\
\hline
\end{tabular}

The IGUI was designed to provide four-way directional control, right, left, up and down. A down command takes the user down a level and the up command brings the user back up a level. However, when operating the IGUI at the top level an 'up' command exits the application. When using a BCI paradigm, several unintended exits due to miss-classifications are expected due to this design. The effects of this can be mitigated by using other input modalities or hybrid combinations. In order to provide more intuitive navigation, an eye tracking system was implemented whereby a user could select a menu item by navigating to it with their gaze and dwelling on it as the selection criteria. Essentially, this approach attempts to simulate a more natural input method where the user's gaze acts as the cursor control and the dwell time simulates the mouse click. The Tobii X60 eye tracker was incorporated as the input modality, which offers high performance but is an expensive product (greater than 10K Euro). More recently, however, the EyeTribe Tracker has been incorporated as an alternative input modality because it is available at a much lower cost (less than 200 Euro), and yet retains sufficient accuracy for this application. At first glance the eye tracking-only system appeared to significantly outperform the BCI-only system in terms of accuracy. After extensive testing, however, a number of false positives were detected, especially when a user paused to read or think. This is a recognized limitation of eye tracking technology and reduces the reliability and of course usability of the IGUI. 
The next iteration of the IGUI was developed to replace the dwell time component with a Brain-Neuronal Computer Interaction (BNCI) approach. BNCI includes devices that monitor additional physiological signals; not only from signals acquired directly from the brain, but from other measures of nervous activity, such as Electrooculography (EoG), Electromyography (EMG) or heart rate. By implementing the Emotiv EPOC BCI device to acquire EEG activity, it was possible to replace the dwell time with a teeth clench/grind component; as a proxy for an EEG feature. The EPOC was employed to extract the representative features from scalp locations AF3, AF4, F3, F4, F8, F7, FC5, FC6, T7, T8, P7, P8, O1, and O2. The following sections of the paper cover details of the protocol and provide an analysis of the results for each approach.

\section{Methods}

For each experiment the same stimulation was employed, which consisted of three tasks to be completed by operation of the IGUI. The first represents a domotic task; the second an entertainment task and the third a communication task. The task details are as follows:

Task 1 domotic control: turn on the lamp (Critical Path = 13). For successful completion, each user was required to navigate to the Dining Room icon, select it and then navigate to the Lamp icon, select it and return to the Back Garden icon. The shortest series of sequential operations (i.e. the least commands for successful completion) is: Right-Right-Right-Right-Down-RightRight-Down(Lamp on)-Up-Left-Left-Left-Left.

Task 2 entertainment: multimedia control (Critical Path = 25). For successful completion, each user was required to navigate to the Living Room icon, select it, navigate to the Home Media icon, select it, navigate to the Home Cinema icon, select it, navigate to a video icon, select it (play video), return to the Home Media icon, navigate to the Controls icon, select it, navigate to the Stop icon, select it (stop video) and return to the Back Garden icon at the top level. The shortest series of sequential operations is: Left-Left-Left-Down-LeftDown-Right-Right-Down-Right-Right-Right-Down(Play)-Up-Left-Left-Down-Right-Down(Stop)-Up-Up-Up-Right-Right-Right.

Task 3 communication: indicate hunger (Critical Path = 7). For successful completion, each user was required to navigate to the Talk icon, select it, navigate to the Eat icon, select it and return to the Back Garden icon. The shortest series of sequential operations is: Left-Down-Left-Left-Down(Eat)-UpRight.

The sequence of commands enacted for Task 1 (a total of 13 individual commands), for a representative subject, is represented in Table 2. Note that the IGUI must be able to deal with repeated correct commands, due to the variable nature of the subject interaction. It does this by dampening the responsiveness of the interaction. Figure 2 shows a subject enacting Task 1 using the hybrid approach. The IGUI is being operated using the Emotiv EPOC and the EyeTribe Tracker as collaborative input modalities. 
Table 2. Sequence of commands enacted for Task 1 for a representative subject. The subject successfully navigated around the interface and turned on the light. They have completed a total of 13 commands but some commands were repeated and disregarded to slow the responsiveness of the application.

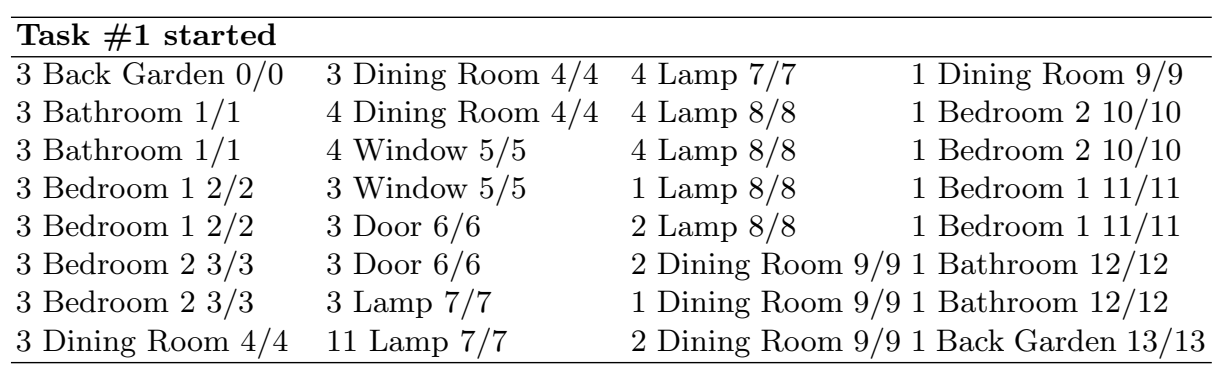

N.B. Task 1 finished with 13 successful commands and 0 invalid commands as repeated commands were disregarded (e.g. command one was to select the bathroom icon, which was activated twice but only executed once). The subject achieved an accuracy $100 \%$ with an average ITR of $16.96 \mathrm{bits} / \mathrm{min}$

\section{Results}

In BCI it is common to measure performance by calculating ITR in bits $/ \mathrm{min}$, which takes into account values for both accuracy and time. According to the literature, traditional BCI systems generally achieved an ITR between 10-25 bits/min [1] whereas conventional input devices such as a mouse and keyboard have been known to achieve an ITR in excess of $300 \mathrm{bits} / \mathrm{min}$ [11]. More recent SSVEP-based BCI systems, which use gel electrodes, have managed to achieve an ITR of at least $60 \mathrm{bits} / \mathrm{min},[12]$. ITR as defined in [13] is shown below:

$$
I T R=\left(\log _{2} M+P \log _{2} P+(1-P) \log _{2}[(1-P) /(M-1)]\right) *(60 / T)
$$

where $\mathrm{M}$ is the number of choices, $\mathrm{P}$ is the accuracy of target detection, and $\mathrm{T}$ (seconds/selection) is the average time for a selection. This metric can be used to evaluate the performance of the input modalities and compare different experiments. In the BRAIN project motor imagery and SSVEP were used to navigate the IGUI. Kus et al. [14] computed an ITR of 12.77 bits/min for a 3-way motor imagery (ERD/ERS) selection task. SSVEP data was then recorded from a control group $(\mathrm{N}=23)$, which incorporated the TMSi amplifier and EasyCap for signal acquisition, the SSVEP paradigm as the control mechanism, screen-mounted LEDs for stimulation and the same three tasks, domotic control, entertainment, and communication by iconography. Table 3 presents the results of the data analysis. An important point to note is that when operating the IGUI at the top level an 'up' command exited the application and as such, several unintended exits due to miss-classifications were categorized as inconclusive. In addition, the '-' symbol in the table indicates that a subject attempted to perform the 


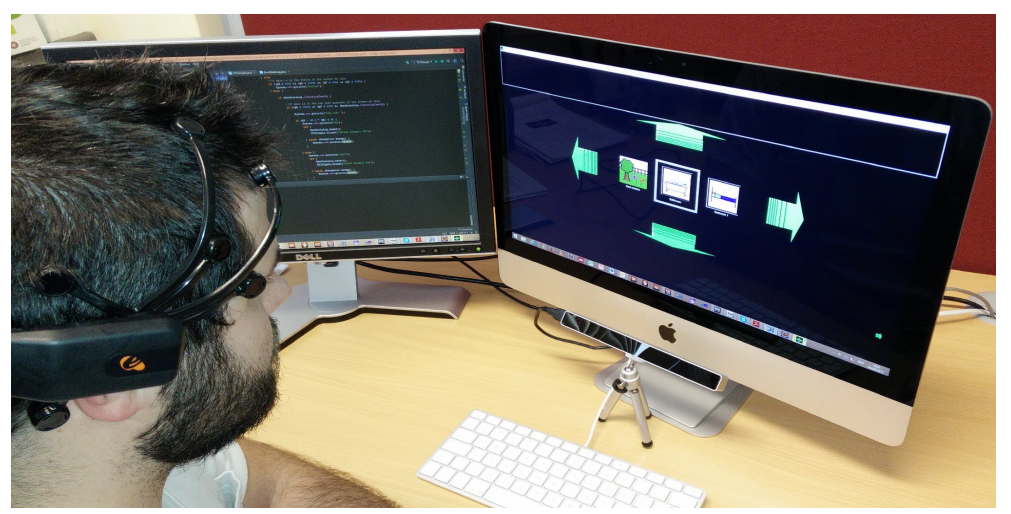

Fig. 2. A subject enacting Task 1 and operating the IGUI while using the EyeTribe Tracker and Emotiv EPOC as collaborative input modalities. The subject has successfully executed a right command thus scrolling the IGUIs menu from the Back Garden icon to the Bathroom icon.

task but could not complete it due to poor performance. Nonetheless, the mean accuracy for all participants that completed all three tasks was $79.2 \% \pm 14.4 \%$ with a mean ITR of $15.23 \pm 7.92 \mathrm{bits} / \mathrm{min}$.

As mentioned previously, an eye tracking component was implemented to provide better navigational control of the IGUI. The third phase of experimentation focused on a control group $(\mathrm{N}=10)$. In this experiment, the SSVEP paradigm was replaced with an eye tracking-only approach. The Tobii X60 Eye Tracker was incorporated as the input modality. When operating the IGUI at the top level an 'up' command still exited the application. As the eye tracker produced a number of false positives, several unintended selections were experienced, which triggered an unintended exit or incorrect command. However, in this case if an unintended exit occurred, the interaction was paused and the experimenter provided a manual override to restart the experiment at the point in which the mistake occurred. If an unintended command occurred, which did not exit the application, the user was required to rectify the error. These values were marked as false positives and were factored in when calculating the accuracy and ITR. Table 4 presents the results using the Tobii X60 eye tracker as the only input modality. Successful completion of all three tasks resulted in a mean accuracy of $78.39 \% \pm 10.21 \%$ and a mean ITR of $194.15 \pm 13.34 \mathrm{bits} / \mathrm{min}$.

The fourth phase of experimentation focused on a further control group of 10 participants. In this experiment, the Tobii X60 based control mechanism was substituted for an EyeTribe Tracker based control method. Similar to the previous experiment, the EyeTribe Tracker was the only input modality and the same protocol was employed once more. Again a number of false positives were detected, which triggered an incorrect command that needed to be rectified by the user or in the case of an unintended exit, by the experimenter. If an unintended exit was experienced the interaction time was paused and a manual 
Table 3. Accuracy and Information Transfer Rate from SSVEP data collected for 23 healthy participants

\begin{tabular}{|c|c|c|c|c|c|c|c|c|}
\hline \multirow[t]{2}{*}{ Subject } & \multicolumn{2}{|c|}{ Domotic } & \multicolumn{2}{|c|}{ Entertainment } & \multicolumn{2}{|c|}{ Communication } & \multicolumn{2}{|c|}{ All tasks } \\
\hline & $\begin{array}{l}\mathrm{ACC} \\
(\%)\end{array}$ & $\begin{array}{l}\text { ITR } \\
\text { (bpm) }\end{array}$ & $\begin{array}{l}\mathrm{ACC} \\
(\%)\end{array}$ & $\begin{array}{l}\text { ITR } \\
\text { (bpm) }\end{array}$ & $\begin{array}{l}\text { ACC } \\
(\%)\end{array}$ & $\begin{array}{l}\text { ITR } \\
\text { (bpm) }\end{array}$ & $\begin{array}{l}\text { ACC } \\
(\%)\end{array}$ & $\begin{array}{l}\text { ITR } \\
\text { (bpm) }\end{array}$ \\
\hline$\overline{\mathrm{T} 1}$ & INCON & CLUSIVE & INCOI & CLUSIVE & INCO & CLUSIVE & INCO & CLUSIVE \\
\hline $\mathrm{T} 2$ & INCON & CLUSIVE & INCOI & CLUSIVE & INCO & CLUSIVE & INCO & CLUSIVE \\
\hline T3 & INCON & CLUSIVE & INCOI & CLUSIVE & INCO & CLUSIVE & INCO & CLUSIVE \\
\hline $\mathrm{T} 4$ & INCON & CLUSIVE & INCOI & CLUSIVE & INCO & CLUSIVE & INCO & CLUSIVE \\
\hline T5 & INCON & CLUSIVE & INCOI & CLUSIVE & INCO & CLUSIVE & INCO & CLUSIVE \\
\hline T6 & 73.33 & 8.95 & 60.47 & 6.03 & 66.67 & 9.45 & 66.82 & 8.14 \\
\hline $\mathrm{T} 7$ & - & & - & & - & & - & \\
\hline T8 & ERROI & & ERRO & & ERRO & & ERRC & \\
\hline T9 & INCON & CLUSIVE & INCOI & CLUSIVE & INCO & CLUSIVE & INCO & CLUSIVE \\
\hline T10 & - & & - & & - & & - & \\
\hline $\mathrm{T} 11$ & 68.80 & 9.89 & - & & - & & INSUI & ICIENT \\
\hline $\mathrm{T} 12$ & 52.78 & 5.78 & - & & 66.67 & 10.38 & INSUl & ICIENT \\
\hline $\mathrm{T} 13$ & - & & - & & - & & - & \\
\hline T14 & 83.33 & 25.95 & 90.00 & 21.15 & 100 & 30.38 & 91.11 & 20.06 \\
\hline T15 & 70.59 & 5.53 & - & & - & & INSUI & ICIENT \\
\hline T16 & INCON & CLUSIVE & INCOI & CLUSIVE & INCO & CLUSIVE & INCO & CLUSIVE \\
\hline $\mathrm{T} 17$ & 100 & 36.23 & 93.33 & 18.57 & 88.89 & 21.69 & 94.07 & 25.5 \\
\hline T18 & 45.16 & 1.82 & 65.38 & 6.56 & 70.00 & 8.10 & 60.18 & 5.49 \\
\hline T19 & - & & - & & - & & - & \\
\hline T20 & 90.00 & 23.70 & 91.67 & 17.23 & 83.33 & 24.06 & 88.33 & 21.66 \\
\hline $\mathrm{T} 21$ & INCON & CLUSIVE & INCOI & CLUSIVE & INCO & CLUSIVE & INCO & CLUSIVE \\
\hline $\mathrm{T} 22$ & 86.67 & 8.37 & - & & - & & INSUI & ICIENT \\
\hline T23 & 100 & 28.44 & 68.97 & 6.64 & 100 & 18.68 & 89.66 & 17.92 \\
\hline Mean: & 77.00 & 15.47 & 78.30 & 12.70 & 82.22 & 17.53 & 79.17 & 15.23 \\
\hline $\mathbf{S D}( \pm):$ & 18.51 & 11.98 & 14.92 & 7.00 & 14.78 & 8.48 & 14.38 & 7.92 \\
\hline
\end{tabular}

override provided to return to the point when the unintended exit occurred. All unintended commands were counted as false positives and commands to rectify a mistake were counted as true positives, since they were intended selections. These values were factored in when calculating accuracy and ITR. Completion of all three tasks for all users equates to a mean accuracy of $93.81 \% \pm 4.59 \%$ and a mean ITR of $164.97 \pm 22.88 \mathrm{bits} / \mathrm{min}$.

The final phase of experimentation included the same control group as the previous experiment $(\mathrm{N}=10)$, which used the EyeTribe Tracker only. In this phase, the Emotiv EPOC was implemented to function collaboratively with the EyeTribe Tracker thus providing multimodal interaction. The same protocol was used once more and a significantly reduced number of false positives were detected. As a result there were no unintended exits experienced by any participant at any time. However, unintended commands still needed to be rectified by the user. In addition, the hybrid system also experienced a rate of false negatives, 
Table 4. Accuracy and Information Transfer Rate data collected for 10 healthy participants using the Tobii X60 as the only input modality

\begin{tabular}{|c|c|c|c|c|c|c|c|c|}
\hline$\overline{\text { Subject }}$ & Domo & & Enter & inment & Com & Inication & $\overline{\text { All } t}$ & \\
\hline & $\begin{array}{l}\mathrm{ACC} \\
(\%)\end{array}$ & $\begin{array}{l}\begin{array}{l}\text { ITR } \\
(\mathrm{bpm})\end{array}\end{array}$ & $\begin{array}{l}\text { ACC } \\
(\%)\end{array}$ & $\begin{array}{l}\begin{array}{l}\text { ITR } \\
\text { (bpm) }\end{array}\end{array}$ & $\begin{array}{l}\text { ACC } \\
(\%)\end{array}$ & $\begin{array}{l}\text { ITR } \\
\text { (bpm) }\end{array}$ & $\begin{array}{l}\mathrm{ACC} \\
(\%)\end{array}$ & $\begin{array}{l}\text { ITR } \\
\text { (bpm) }\end{array}$ \\
\hline$\overline{\text { UU101 }}$ & 36.11 & 153.43 & 58.70 & 161.20 & 84.62 & 129.01 & 59.81 & 189.58 \\
\hline UU102 & 81.25 & 132.32 & 61.36 & 185 & 84.62 & 158.78 & 75.74 & 207.57 \\
\hline UU103 & 100 & 138. & 90.0 & 173 & 100 & 150.13 & 96.67 & 198.56 \\
\hline UU104 & 86.67 & 151.34 & 87.10 & 128.01 & 91.67 & 132.95 & 88.48 & 166.19 \\
\hline UU105 & 76.47 & 175.17 & 84.38 & 178.11 & 84.62 & 147.44 & 81.81 & 212.42 \\
\hline UU106 & 81.25 & 147.02 & 67.50 & 167.40 & 78.57 & 152.38 & 75.77 & 198.71 \\
\hline UU1 & 81.25 & 160. & 58.70 & 163 & 73.33 & 157.52 & 71.09 & 199.77 \\
\hline J108 & 72.22 & 126.19 & 62.79 & 163.31 & 78.57 & 122.75 & 71.19 & 181.22 \\
\hline UU109 & 86.67 & 124.85 & 62.79 & 169.85 & 100 & 128.69 & 83.15 & 188.91 \\
\hline UU110 & 92.86 & 134.61 & 69.23 & 168.45 & 78.57 & 163.66 & 80.22 & 198.53 \\
\hline Mean: & 79.48 & 144.4 & 70.26 & 165.78 & 85.46 & 144.33 & 78.39 & 194.15 \\
\hline $\mathbf{S D}( \pm):$ & 17.18 & 16.07 & 12.20 & 15.16 & 9.15 & 14.68 & 10.21 & 13.34 \\
\hline
\end{tabular}

which were not present during the eye tracking-only experiments. A false negative is more tolerable as it does not trigger an incorrect command. Nevertheless, such errors are factored in when calculating the accuracy and ITR. For all tasks the mean accuracy was $96.04 \% \pm 3.56 \%$ and the mean ITR was $207.41 \mathrm{bits} / \mathrm{min}$.

In our tests, the hybrid system achieved the highest performance in terms of both accuracy and ITR. It is difficult to determine which eye tracking system had the better performance since the Tobii X60 system had lower accuracy but a higher ITR than the EyeTribe system. However, there exists a tradeoff between accuracy and ITR, which means that a more accurate system may report a lower ITR since unintended selections need to be rectified by an additional intended selection; thereby increasing the overall bit rate.

\section{Discussion}

Previous research studies have focused largely on single paradigms in order to facilitate control using BCI systems. These studies have accuracy, performance and reliability limitations [10]. The combination of different input modalities may improve upon these results, thus providing a greater level of control [10]. For instance, an active BCI paradigm, such as SSVEP and/or Motor Imagery (MI), can deliver a method of direct control, whilst a passive BCI paradigm, using error potentials, for example, can potentially provide implicit control [15]. Such a system could benefit from reinforced decisions while autonomously correcting false positive no-control states, thereby improving upon the performance that the use of a single paradigm can offer. There is still a gap between what an acceptable accuracy for a usable system is and what can be readily achieved outside the specialist laboratory [16]. Indeed there is a further difference in the performance 
Table 5. Accuracy and Information Transfer Rate data collected for 10 healthy participants using the EyeTribe Tracker as the only input modality

\begin{tabular}{|c|c|c|c|c|c|c|c|c|}
\hline Subject & Domo & & Entert & inment & Comr & inication & All ta & \\
\hline & $\begin{array}{l}\text { ACC } \\
(\%)\end{array}$ & $\begin{array}{l}\text { ITR } \\
\text { (bpm) }\end{array}$ & $\begin{array}{l}\text { ACC } \\
(\%)\end{array}$ & $\begin{array}{l}\text { ITR } \\
(\mathrm{bpm})\end{array}$ & $\begin{array}{l}\text { ACC } \\
(\%)\end{array}$ & $\begin{array}{l}\text { ITR } \\
\text { (bpm) }\end{array}$ & $\begin{array}{l}\text { ACC } \\
(\%)\end{array}$ & $\begin{array}{l}\text { ITR } \\
\text { (bpm) }\end{array}$ \\
\hline$\overline{\text { UU201 }}$ & 100 & 65.39 & 96.15 & 137.11 & 100 & 106.27 & 98.72 & 127.55 \\
\hline UU202 & 100 & 143.43 & 100 & 160 & 100 & 118.77 & 100 & 183.89 \\
\hline UU203 & 93.33 & 135.50 & 100 & 142.37 & 80.00 & 131.10 & 91.11 & 170.75 \\
\hline UU204 & 76.47 & 87.59 & 93.10 & 173.12 & 100 & 87.79 & 89.86 & 153.85 \\
\hline UU205 & 93.33 & 114.42 & 100 & 132 & 100 & 74.78 & 97.78 & 143.71 \\
\hline UU206 & 93.33 & 107.27 & 100 & 14 & 88.8 & 125.17 & 94.07 & 159.85 \\
\hline UU207 & 87.50 & 164.74 & 92.59 & 151 & 88.89 & 146.04 & 89.66 & 191.28 \\
\hline UU208 & 88.24 & 163.45 & 87.10 & 173.09 & 88.89 & 138.35 & 88.07 & 203.85 \\
\hline UU209 & 100 & 127.04 & 96.30 & 136 & 100 & 96.15 & 98.77 & 157.07 \\
\hline UU210 & 88.24 & 122.59 & 93.10 & 139.75 & 88.89 & 101.10 & 90.08 & 157.85 \\
\hline Mean: & 92.04 & 123.14 & 95.83 & 149.36 & 93.56 & 112.55 & 93.81 & 164.97 \\
\hline $\mathbf{S D}( \pm):$ & 7.36 & 31.38 & 4.37 & 14.91 & 7.29 & 23.11 & 4.59 & 22.88 \\
\hline
\end{tabular}

of such systems when comparing users suffering from brain injury and control subjects [15]. The motivation for this research is that solutions based on a hBCI architecture could harness features from complementary technologies [6] thereby closing these gaps.

Both eye tracking systems investigated outperformed pure BCI systems incorporating SSVEP and ERD/S approaches in terms of ITR. However eye tracking as a technology suffers from false positives when the user is in a 'no control' state. Hence there is a need for a hybrid system, where the user can 'initiate' a command in a predictable manner. At present the hBCI uses a teeth clench as a 'proxy' for an EEG controlled switch. This is for pragmatic reasons because the Emotiv suite comes preloaded with classification algorithms for teeth clench. Furthermore, eye tracking technology, which can aid severely disabled individuals by providing them with a level of control, also experiences its own set of limitations. In general, eye tracking uses both infra-red signals and reflected light to determine the trajectory of a user's gaze. This technology is known to provide efficient cursor control but suffers from intended selection restrictions, an area of significant attention in BCI. Earlier research studies have focused on the frequency of blinks and dwell times as the selection criteria, however, the results are not yet promising [17], thus increasing the demand for further innovation.

Subsequently, the collaboration of both BCI and eye tracking as input modalities may mitigate the individual limitations of each and provide a more natural and usable hybrid system. In 2010 Lee et al. [18] proposed a method to combine BCI and Eye Tracking for 3D interaction using a bespoke head-mounted eye tracking device with attached EEG sensors. According to the experimental results, the feasibility of the proposed 3D interaction method using eye tracking and BCI was confirmed [18]. The Tobii X60 eye tracker is able to provide 
Table 6. Accuracy and Information Transfer Rate data collected for 10 healthy participants using the EyeTribe Tracker and Emotiv EPOC as collaborative input modalities

\begin{tabular}{|c|c|c|c|c|c|c|c|c|}
\hline Subject & Domc & & Enter & inment & Com & inication & All ta & \\
\hline & $\begin{array}{l}\text { ACC } \\
(\%)\end{array}$ & $\begin{array}{l}\text { ITR } \\
(\mathrm{bpm})\end{array}$ & $\begin{array}{l}\text { ACC } \\
(\%)\end{array}$ & $\begin{array}{l}\text { ITR } \\
\text { (bpm) }\end{array}$ & $\begin{array}{l}\text { ACC } \\
(\%)\end{array}$ & $\begin{array}{l}\text { ITR } \\
\text { (bpm) }\end{array}$ & $\begin{array}{l}\text { ACC } \\
(\%)\end{array}$ & $\begin{array}{l}\text { ITR } \\
(\mathrm{bpm})\end{array}$ \\
\hline UU201 & 92.86 & 172.98 & 100 & 204.08 & 77.78 & 151.20 & 90.21 & 225.41 \\
\hline UU202 & 100 & 195.73 & 100 & 153.66 & 100 & 179.52 & 100 & 202.86 \\
\hline UU203 & 100 & 158.70 & 96.15 & 187.24 & 100 & 134.64 & 98.72 & 208.84 \\
\hline UU204 & 92.86 & 183.15 & 89.29 & 214.30 & 100 & 134.64 & 94.05 & 235.99 \\
\hline UU205 & 86.67 & 120.09 & 89.66 & 132.76 & 100 & 122.40 & 92.11 & 156.54 \\
\hline UU206 & 92.86 & 163.88 & 100 & 192.07 & 100 & 107.71 & 97.62 & 204.83 \\
\hline UU207 & 88.24 & 180.60 & 89.29 & 157.78 & 100 & 122.40 & 92.51 & 195.36 \\
\hline UU208 & 100 & 163.11 & 100 & 207.32 & 100 & 141.73 & 100 & 223.49 \\
\hline UU209 & 100 & 150.56 & 96.15 & 179.75 & 100 & 128.23 & 98.72 & 199.56 \\
\hline UU210 & 100 & 167.77 & 89.29 & 199.42 & 100 & 134.64 & 96.43 & 221.26 \\
\hline Mean: & 95.35 & 165.66 & 94.98 & 182.84 & 97.78 & 135.71 & 96.04 & 207.41 \\
\hline $\mathbf{S D}( \pm):$ & 5.30 & 20.69 & 5.04 & 26.70 & 7.03 & 19.42 & 3.56 & 22.15 \\
\hline
\end{tabular}

consistent and reliable control, albeit with some false positives. However it is expensive and therefore is only applicable to laboratory based testing. In addition the Application Programming Interface (API) is not open source and as such, this limits its value as a research tool. By combining the Eye Tribe eye tracker with the EPOC (using a teeth clench for selection), the ITR increased to the performance of the much more expensive eye tracker. We aim for an open source system that incorporates inexpensive and commercially available eye tracking and BCI hardware.

The performance improvement, which is evident from the results in table 6 compared with the results in tables 4 and 5 , shows that the tested hybrid system outperforms eye tracking only based approaches in terms of accuracy and ITR. It also outperforms the SSVEP only system. When questioned all users reported that the hybrid system provided greater control as it facilitated the ability to pause, think and even talk in between attempting to execute commands. Unlike the eye tracking only approaches the hybrid did not issue false positives, which are intolerable when controlling events in a smart environment. In this case unintentional commands, which execute events such as opening and closing doors, switching lights on and off, flushing the toilet, opening and closing the garden gate, interacting with food preparation devices, and security systems could create a state of bedlam. Seemingly, the burden of montage of an EEG devices is therefore less severe.

\section{Conclusion}

In this work we have shown the feasibility of using collaborative input modalities based on a hybrid BCI approach. Both of the devices utilized are cost-effective, 
highly usable and sufficiently accurate, which makes them particularly suited for deployment outside of laboratory conditions and into 'the wild'. A benefit of the hybrid approach lies in the system ability to interact only when desired by the user. Each user reported full control of the system, i.e. they were able to pause, think, read and talk in between each task without controlling the system, unintentionally. We have also shown that the fusion of BNCI and eye tracking technology reaches the performance of conventional BCI systems and eye tracking-only approaches. Our objective is to investigate how we can replace the BNCI component with a 'true BCI' paradigm, such as SSVEP. It is of importance to maintain high ITRs while maintaining an efficient and robust system that can be widely deployed. Although such components may not meet the performance of research-grade devices, in our tests the Eye Tribe proved sufficiently accurate to provide the desired level of control. By utilizing devices such as the Eye Tribe tracker and the Emotiv EPOC collaboratively, we believe that it is possible to deploy this system outside of the laboratory.

In the next phase of work, low frequency SSVEP stimulation will be incorporated into the IGUI using on screen pattern reversal positioned at each control point. Four different frequencies will be implemented in a collaborative manner with eye tracking. Each selection can only be made if two conditions are true; the gaze based co-ordinates are in an appropriate area and a frequency within a pre-defined threshold is detected in the EEG. Signal acquisition, processing, and classification will be handled within the OpenVibe environment and VRPN will facilitate fusion between the Eye Tribe Tracker and EEG. At first the EEG signal will be acquired using g.Tec's g.USBamp which, is also supported within the current version of OpenVibe. Experimentation will focus on a control group of 20 subjects who will complete the same tasks twice, once using the EPOC and once using the g.Tec device. A comparative study will contrast the two devices and provide an indication of the difference in usability and performance. A further study in conjunction with the Cedar Foundation will test our system on clients with brain injury to establish it's utility as a collaborative assistive technology. A number of challenges may arise: 1) differentiating between frequency bands 2 ) poor performance by brain injury subjects; and 3) inconsistent frequencies caused by the refresh rate.

\section{References}

1. Wolpaw, J.R., Birbaumer, N., Mcfarland, D.J., Pfurtscheller, G., Vaughan, T.M.: Brain computer interfaces for communication and control. Clinical Neurophysiology 113(6) (2002) 767-791

2. Coyle, D., Principe, J., Lotte, F., Nijholt, A.: Guest Editorial: Brain/neuronal Computer game interfaces and interaction. IEEE Transactions on Computational Intelligence and AI in Games 5(2) (June 2013) 77-81

3. Lotte, F., Faller, J., Guger, C.: Combining BCI with Virtual Reality: Towards New Applications and Improved BCI. In: Proceedings of the 6th International Conference on Foundations of Digital Games. (2013) 1-24 
4. Volosyak, I., Valbuena, D., Malechka, T., Peuscher, J., Gräser, A.: Brain-computer interface using water-based electrodes. Journal of neural engineering 7(6) (December 2010) 066007

5. McFarland, D.J., Miner, L.a., Vaughan, T.M., Wolpaw, J.R.: Mu and beta rhythm topographies during motor imagery and actual movements. Brain topography 12(3) (January 2000) 177-86

6. Mccullagh, P., Galway, L., Lightbody, G.: Investigation into a Mixed Hybrid Using SSVEP and Eye Gaze for Optimising User Interaction within a Virtual Environment. Universal Access in Human-Computer Interaction. Design Methods, Tools, and Interaction Techniques for eInclusion 8009 (2013) 530-539

7. Allison, B.Z., Brunner, C., Kaiser, V., Müller-Putz, G.R., Neuper, C., Pfurtscheller, G.: Toward a hybrid brain-computer interface based on imagined movement and visual attention. Journal of neural engineering 7(2) (April 2010) 26007

8. Thomson, E., Mathews, S., Todd, D., McCullagh, P., Ware, M., Mulvenna, M., Martin, S.: Developing brain computer interfaces with rapid automated interfaces for non experts. Gerontechnology 9(2) (April 2010) 4017

9. Todd, D.a., McCullagh, P.J., Mulvenna, M.D., Lightbody, G.: Investigating the use of brain-computer interaction to facilitate creativity. Proceedings of the 3rd Augmented Human International Conference on - AH '12 (2012) 1-8

10. Ware, M.P., McCullagh, P.J., McRoberts, A., Lightbody, G., Nugent, C., McAllister, G., Mulvenna, M.D., Thomson, E., Martin, S.: Contrasting levels of accuracy in command interaction sequences for a domestic brain-computer interface using SSVEP. In: Biomedical Engineering Conference (CIBEC), 2010 5th Cairo International, Ieee (December 2010) 18-21

11. Krepki, R., Blankertz, B., Curio, G., Müller, K.R.: The Berlin Brain-Computer Interface (BBCI) - Towards a new communication channel for online control in gaming applications. Multimedia Tools and Applications 33(1) (2007) 73-90

12. Chen, X., Chen, Z., Gao, S., Gao, X.: A high-ITR SSVEP-based BCI speller. Taylor \& Francis: Brain Computer Interfaces 1(3 \& 4) (2014) 181-191

13. Gao, S., Wang, Y., Gao, X., Hong, B.: Visual and auditory brain-computer interfaces. IEEE transactions on bio-medical engineering 61(5) (May 2014) 1436-47

14. Kus, R., Valbuena, D., Zygierewicz, J., Malechka, T., Graeser, A., Durka, P.: Asynchronous BCI based on motor imagery with automated calibration and neurofeedback training. IEEE Transactions on Neural Systems and Rehabilitation Engineering 20 (2012) 823-835

15. George, L., Lécuyer, A.: An overview of research on passive brain-computer interfaces for implicit human-computer interaction. In: International Conference on Applied Bionics and Biomechanics ICABB 2010. (2010)

16. Allison, B., Luth, T., Valbuena, D., Teymourian, A., Volosyak, I., Graser, A.: BCI Demographics : How Many ( and What Kinds of ) People Can Use an SSVEP BCI? IEEE Transactions on Neural Systems and Rehabilitation Engineering 18(2) (2010) 107-116

17. Lightbody, G., Ware, M., McCullagh, P., Mulvenna, M., Thomson, E., Martin, S., Todd, D., Medina, V.C., Martinez, S.C.: A user centred approach for developing Brain-Computer Interfaces. In: Proceedings of the 4th International ICST Conference on Pervasive Computing Technologies for Healthcare, Ieee (2010)

18. Lee, E.C., Woo, J.C., Kim, J.H., Whang, M., Park, K.R.: A brain-computer interface method combined with eye tracking for 3D interaction. Journal of Neuroscience Methods 190 (2010) 289-298 